\title{
Contrast Enhancement using Improved Adaptive Gamma Correction With Weighting Distribution Technique
}

\author{
Seema Rani \\ Research Scholar Computer Engineering \\ Department \\ Yadavindra College of Engineering Talwandi sabo, \\ Bathinda, India
}

\author{
Manoj Kumar \\ Assistant Professor Computer Engineering \\ Department \\ Yadavindra College of Engineering Talwandi sabo, \\ Bathinda, India
}

\begin{abstract}
One of the important techniques in digital image processing is to enhance images. Contrast enhancement is a method that is used to enhance images for viewing process or for further analysis of images. Main idea behind contrast enhancement techniques is to increase contrast and to preserve original brightness of images. In this paper a contrast enhancement technique is proposed that first segments histogram of image recursively and then applies Adaptive Gamma Correction with Weighting Distribution (AGCWD) Technique. The proposed technique is basically an improvement over AGCWD technique and aims to get better contrast enhancement and brightness preservation than AGCWD technique.
\end{abstract}

\section{General Terms}

Image Enhancement, Contrast Enhancement.

\section{Keywords}

Histogram Equalization, Recursive Segmentation, Histogram Modification, Gamma Correction, Weighting Distribution.

\section{INTRODUCTION}

The image enhancement is one of the significant techniques in digital image processing. It has an important role in various fields where images are to be understood and analyzed. Image enhancement is done on an image to improve its visual effects and quality or to make it more appropriate for further processing by another application. An image can have low contrast or bad quality due to a number of reasons like poor quality of imaging device, adverse external conditions at the time of image acquisition and many more. The contrast enhancement is one of the commonly used image enhancement method [1].

Histogram equalization is the traditional technique for contrast enhancement. It basically maps gray levels based on probability distribution of input image [2]. But image obtained by this method can produce undesirable effects in image and also original brightness of image is not preserved. Histogram equalization technique redistributes probability densities. Adaptive Gamma Correction with Weighting Distribution (AGCWD) technique is based on histogram modification method [3]. This technique combines both gamma correction and histogram equalization techniques. Gamma correction is a transform based histogram modification technique that uses a varying parameter $\gamma$ (gamma). Gamma correction method had problem that unvaried modification results for every image because a predefined value was used for all images. Histogram equalization had problem of under enhancement and over enhancement. So the AGCWD technique removed disadvantages of both gamma correction and Histogram Equalization techniques by combining both techniques and using a weighting function. In this technique gamma correction is applied using normalized cumulative density function (cdf).

The AGCWD technique effectively enhances images. To further improve this technique to get better contrast enhancement and better brightness preservation an improvement is proposed in this paper. Improvement proposed is based on recursive segmentation of histogram.

\section{LITERATURE REVIEW}

Traditional Histogram Equalization method may change the original brightness and can deteriorate visual quality of image. To solve these problems Y.T. Kim proposed Brightness preserving Bi-Histogram Equalization method (BBHE) that equalizes two sub histograms produced by histogram separation techniques and calculates mean intensity as threshold [4]. Another technique Dualistic Sub Image Histogram Equalization (DSIHE) uses median as threshold to separate histograms instead of mean [5]. The Minimum Mean Brightness Error BiHistogram Equalization (MMBEBHE) has the feature of minimizing the difference between input and output image's mean. MMBEBHE can preserve brightness better than BBHE and DSIHE. But MMBEBHE has limitation of high computational complexity [6]. Thus a generalization of BBHE referred to as Recursive Mean-Separate Histogram Equalization (RMSHE) was introduced. RMSHE was featured with scalable brightness preservation [7]. The Brightness Preserving Histogram Equalization with Maximum Entropy (BPHEME) method maximizes the entropy by the variational approach under the constraints that the mean brightness remains fixed [8]. The Recursive Sub Image Histogram Equalization (RSIHE) technique extends DSIHE by recursively separating histogram and multi-equalizations to solve above problems [9]. But the problems were not effectively solved in spite of its recursive nature and scalable brightness preservation techniques. Another histogram separation technique Recursively Separated and Weighted Histogram Equalization (RSWHE) uses a weighting function to smooth each sub histogram and to effectively solve the mean-shift problem [10]. Renjie He, Sheng Luo, Zhanrong Jing and Yangyu Fan developed a method in which the weighted average of histogram equalization and exponential transformation are combined and the level of the contrast improvement is adjustable by changing the weighting coefficients. The algorithm achieved adjustable contrast enhancement for color images and also decreased the effect of rising intensity on colors of image [1].

A method that combined histogram equalization and gamma correction methods was applied in [11]. But in this technique 
the value for gamma is not adjusted automatically and the value is to be assigned manually. It avoids over enhancement caused by the traditional HE. The CVC (Contextual and Variational Contrast) enhancement technique enhances the contrast of an image using 2D histogram of the input image constructed using mutual relationship between each pixel and its neighboring pixels. But the technique has high computational complexity [12]. A technique for fog removal using Fast Fourier Transformation was also proposed [13]. A technique was proposed in [19] in which contrast is enhanced and adjustments are made according to atmospheric light.

Shih-Chia Huang, Fan-Chieh Cheng and Yi-Sheng Chiu proposed a hybrid HM (histogram modification) method Adaptive Gamma Correction with Weighting Distribution (AGCWD) by combining TGC (Transform based gamma correction) and THE (Traditional histogram equalization) methods. This paper presented an automatic transformation technique that improved the brightness of dimmed images via the gamma correction and probability distribution of grey levels. For enhancement of videos, the technique used temporal information regarding the differences between each frame to reduce computational complexity [3].

\section{ADAPTIVE GAMMA CORRECTION WITH WEIGHTING DISTRIBUTION}

According to AGCWD method in [3], Adaptive gamma correction is formulated in as:

$$
\mathrm{T}(\mathrm{l})=1_{\max }\left(1 / 1_{\max }\right)^{\gamma}=\operatorname{lmax}(1 / 1 \max )^{1-\mathrm{cdf}(\mathrm{l})}
$$

Weighting distribution function is applied as:

$$
\operatorname{Pdf}_{\mathrm{w}}(1)=\operatorname{pdf}_{\max }\left(\operatorname{pdf}(1)-\operatorname{pdf}_{\min } / \operatorname{pdf}_{\max }-\operatorname{pdf}_{\min }\right)^{\alpha}
$$

where $\alpha$ is the adjusted parameter, $\operatorname{pdf}_{\max }$ is the maximum pdf of statistical histogram, and $\mathrm{pdf}_{\min }$ is minimum pdf. Then modified cdf is as:

$$
\operatorname{cdf}_{\mathrm{w}}(1)=\sum_{l=0}^{l \max } \operatorname{Pdfw}(\mathrm{l}) / \sum \mathrm{pdfw}
$$

where the sum of $\operatorname{pdf}_{\mathrm{w}}$ calculated as follows:

$$
\sum \mathrm{pdfw}=\sum_{l=0}^{\operatorname{lmax}} \mathrm{pdfw}(\mathrm{l})
$$

And, gamma for equation (1) is calculated as:

$$
\gamma=1-c d f_{w}(1)
$$

\section{PROPOSED METHOD}

In this section the proposed method for image contrast enhancement is presented. The algorithm is designed to efficiently improve contrast and preserve original image brightness. The proposed method consists of steps as shown in flowchart in figure1.

\subsection{Image acquisition}

The image can be obtained by any digital device like mobile phone, laptop and other cameras. Image can be color or grayscale. A dataset of 10 standard images has been taken, from which 5 are grayscale and 5 are colored images.

\subsection{Histogram of image}

Algorithm is based on histogram equalization technique. So after acquisition of image, histogram of image is obtained. So that further processing can be done on image histogram. Image histogram is obtained by built in function imhist.

\subsection{Recursive segmentation of histogram}

Recursive segmentation of histogram is done based on chosen value for recursion level i.e. r. Based on this value $2^{\mathrm{r}}$ sub histograms will be obtained. Histograms are divided based on the mean of input image. Mean value for a sub histogram is calculated as:

Let $\mathrm{H}^{\mathrm{t}}(\mathrm{X})$ be a segmented histogram over a gray level range $\left[\mathrm{X}_{\mathrm{L}}, \mathrm{X}_{\mathrm{U}}\right]$ at a recursion level $\mathrm{t}(0 \leq \mathrm{t}<\mathrm{r})$. The mean $\mathrm{X}_{\mathrm{m}}{ }^{\mathrm{t}}$ of the sub image histogram $\mathrm{H}^{\mathrm{t}}(\mathrm{x})$ is computed as:

$$
\mathrm{X}_{\mathrm{m}}{ }^{\mathrm{t}}=\sum_{\mathrm{k}=\mathrm{l}}^{\mathrm{u}} \mathrm{k} \cdot \mathrm{p}(\mathrm{k}) / \sum_{\mathrm{k}=\mathrm{l}}^{\mathrm{u}} \mathrm{p}(\mathrm{k})
$$

Based on computed mean $\mathrm{X}_{\mathrm{m}}{ }^{\mathrm{t}}$ the histogram $\mathrm{H}^{\mathrm{t}}(\mathrm{X})$ is divided into two sub histograms $\mathrm{H}^{\mathrm{t}+1} \mathrm{~L}(\mathrm{X})$ and $\mathrm{H}_{\mathrm{U}}^{\mathrm{t}+1}(\mathrm{X})$ for the next recursion level $t+1$ and these are defined over $\left[\mathrm{X}_{\mathrm{L}}, \mathrm{X}_{\mathrm{m}}{ }^{\mathrm{t}}\right]$ and $\left[\mathrm{X}_{\mathrm{m}+1}{ }^{\mathrm{t}}, \mathrm{X}_{\mathrm{U}}\right]$ respectively.

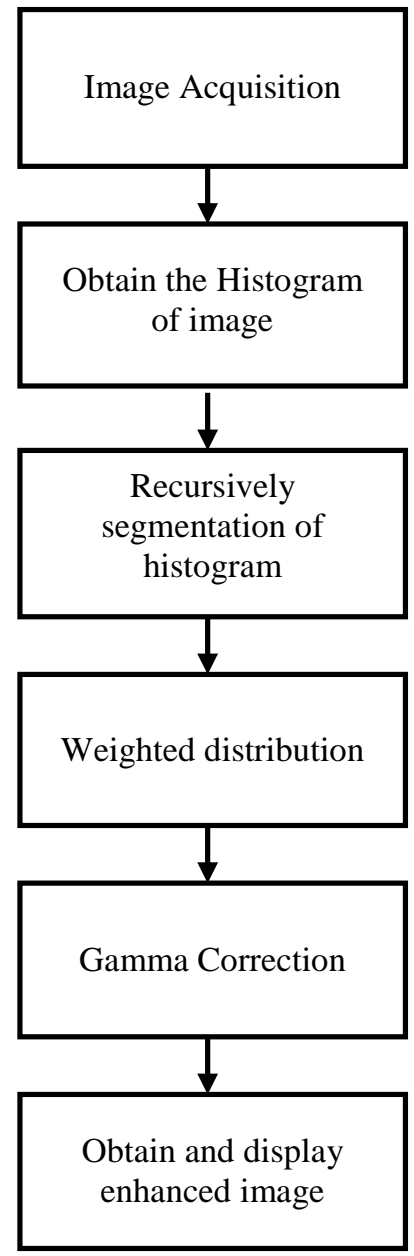

Figure 1: Steps for Implementation of Proposed Technique

\subsection{Weighted distribution}

Weighted distribution is applied so that the image regions with high probability should not get over enhanced and image regions with less probability should not be less enhanced and no loss in important visual details must occur. In this input histogram is modified in the way that less frequent gray levels are given more probabilities or weights. Weighted Pdf is calculated as:

$$
\operatorname{Pdf}_{\mathrm{w}}(1)=\operatorname{pdf}_{\max }\left(\operatorname{pdf}(1)-\operatorname{pdf}_{\min } / \operatorname{pdf}_{\max }-\operatorname{pdf}_{\min }\right)^{\alpha}
$$

where $\alpha$ is the adjusted parameter, $\operatorname{pdf}_{\max }$ is the maximum pdf of statistical histogram, and $\mathrm{pdf}_{\min }$ is minimum pdf. Now modified cdf is as:

$$
\operatorname{cdf}_{\mathrm{w}}(\mathrm{l})=\sum_{l=0}^{\operatorname{lmax}} \operatorname{Pdfw}(\mathrm{l}) / \sum \mathrm{pdfw}
$$


where

$$
\sum p d f w=\sum_{l=0}^{\operatorname{lmax}} \operatorname{pdfw}(\mathrm{l})
$$

\subsection{Gamma correction}

After weighted distribution the sub histograms are mapped to final histogram and gamma correction is applied. In this technique gamma correction is applied using normalized cumulative density function (cdf). Gamma correction is done as:

$$
\mathrm{T}(\mathrm{l})=1_{\max }\left(1 / 1_{\max }\right)^{\gamma}=\operatorname{lmax}(1 / 1 \max )^{1-\operatorname{cdf}(1)}
$$

Where, gamma for equation (32) is calculated as:

$$
\gamma=1-c d f_{w}(1)
$$

Now final image is obtained after gamma correction.

\subsection{Output enhanced image}

Finally the enhanced image is obtained after gamma correction. Now the final output image is tested using various parameters which are discussed in next section.

\section{EXPERIMENTAL RESULTS}

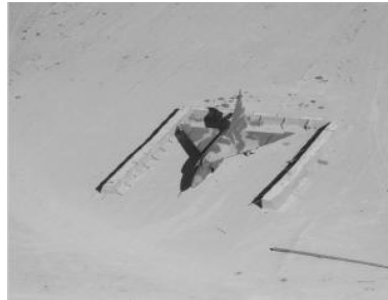

(a) Original Image

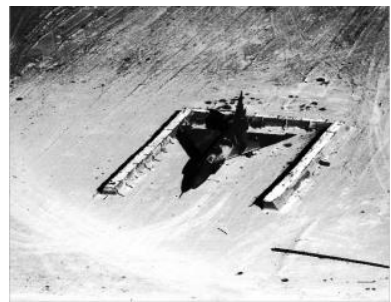

(c) BBHE

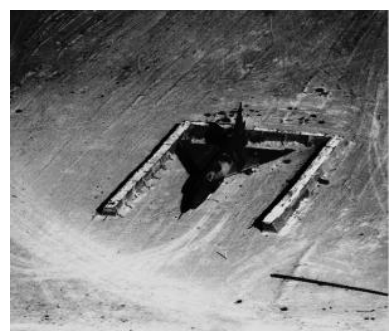

(e) AGCWD

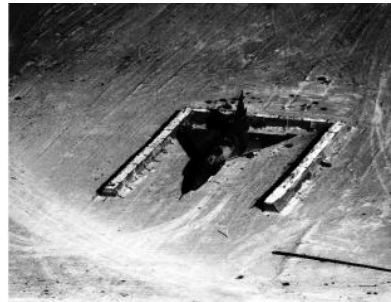

(b) $\mathrm{HE}$

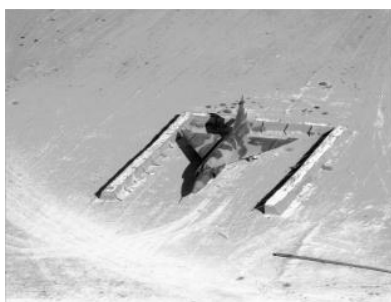

(d) RSWHE

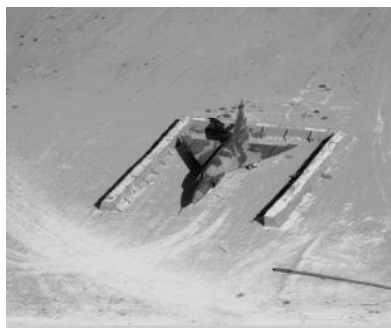

(f) Proposed Technique
Figure 2: War Plane Image (a) Original image (b) HE processing (c) BBHE processing (d) RSWHE processing (e) AGCWD processing (f) Proposed technique

The results of proposed technique are compared with techniques including Histogram equalization (HE), Brightness preserving bi histogram equalization (BBHE) [4], Recursively separated and weighted histogram equalization (RSWHE) [10] and Adaptive gamma correction using weighted distribution (AGCWD) [3] technique. A dataset of 10 images is taken that are previously used in reference papers to compare the results in which 5 are gray scale images and 5 are color images. In this paper result evaluation is shown on 4 images. In which 2 are gray scale images and 2 are color images.

\subsection{Visual assessment}

For visual assessment initially two grayscale images named war plane image [3] and cameraman image [15] are taken. Figure 2 shows enhancement results on war plane image by different techniques. HE technique directly equalizes image, thus results in loss of information as shown in Figure 2(b). BBHE technique equalizes dark and light gray levels separately to solve problem by HE method. But still brightness of image could not be preserved as shown in Figure 2(c). RSWHE technique somewhat equalized image but contrast is not improved effectively as shown in Figure 2(d). Similarly the AGCWD technique cannot effectively preserve original brightness of image as shown in Figure 2(e). It can be directly observed from Figure 2 By viewing. It is clearly seen in figure 2(f) that the enhancement made by proposed technique is natural and better as compared to other techniques. Also the technique has better preserved original brightness of image.

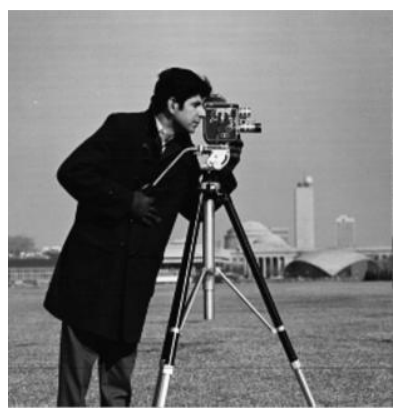

(a) Original Image

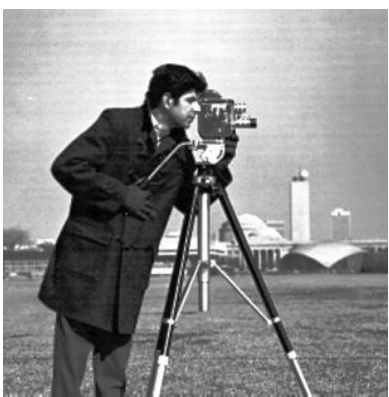

(c) BBHE

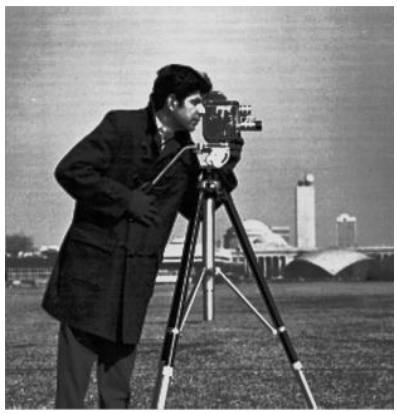

(e) AGCWD

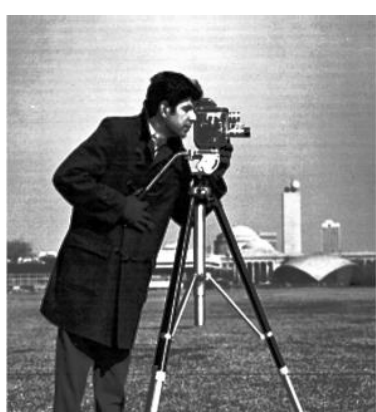

(b) $\mathrm{HE}$

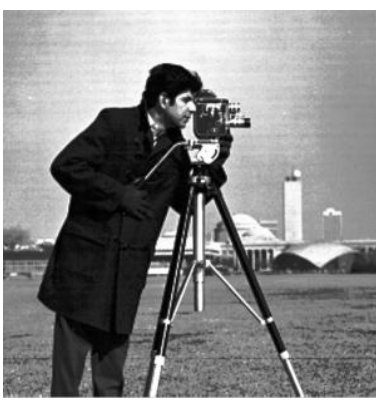

(d) RSWHE

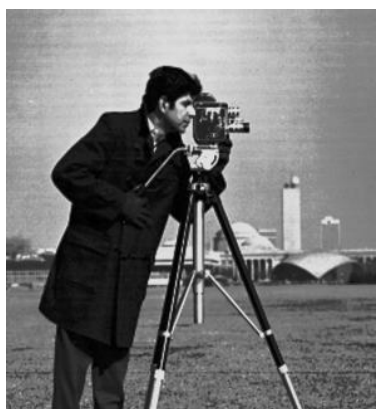

(f) Proposed Technique
Figure 3: Cameraman Image (a) Original image (b) HE processing (c) BBHE processing (d) RSWHE processing (e) AGCWD processing (f) Proposed technique 
Figure 3 shows enhancement results on cameraman image by different techniques. Proposed technique enhances contrast more efficiently and naturally. Histogram equalization technique can result in unnatural enhancement; it can be seen in the figure 3(b). BBHE and AGCWD technique cannot preserve brightness effectively as shown in Figure 3(c) and Figure 3(e) and the contrast enhancement by RSWHE technique is not effective as shown in Figure 3(d). Proposed technique preserves brightness better than other techniques and contrast enhancement is done effectively as shown in Figure 3(f).

For further evaluation, two color images named house image [10] and F-16 image [4] are taken. Enhancement is performed on color images using RGB color map. Figure 4 shows enhancement results on house image by different techniques. Some adverse effect on result images can be seen from Figure 4(b)-4(e). The sky color in house image is distorted in these figures. AGCWD technique fails to retain original brightness of image as shown in Figure 4(e). Proposed technique produced image with natural and acceptable brightness as in Figure 4(f).

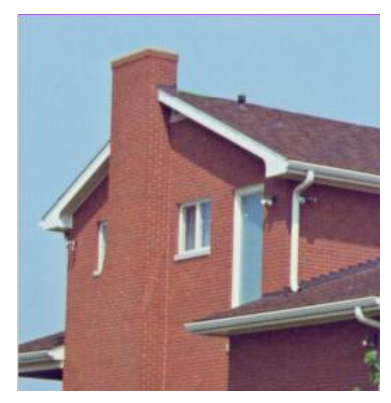

(a) Original Image

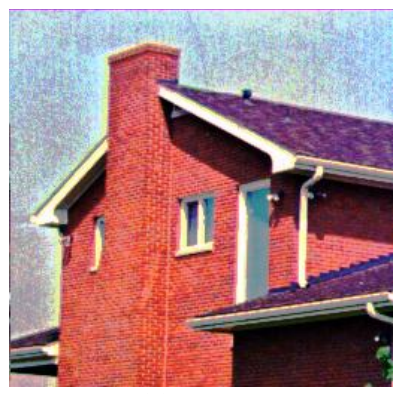

(c) BBHE

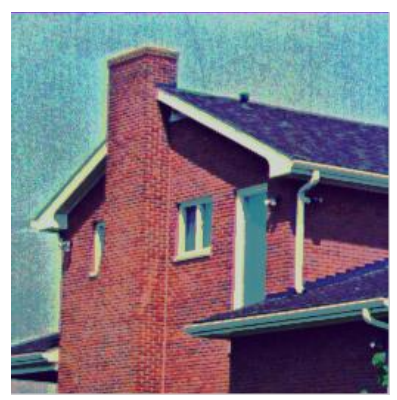

(e) AGCWD

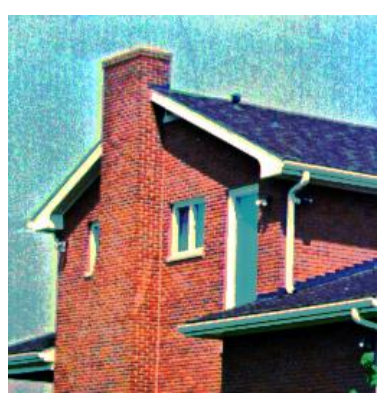

(b) HE

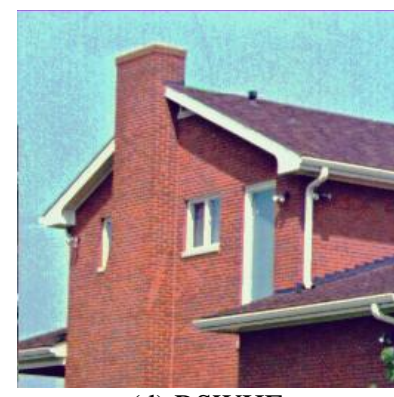

(d) RSWHE

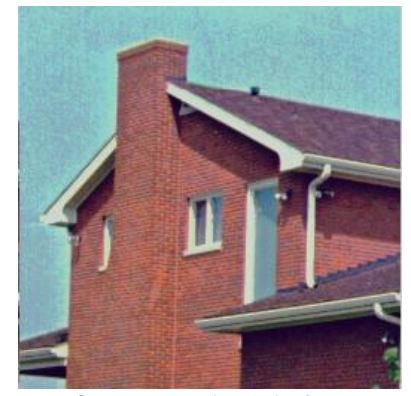

(f) Proposed Technique
Figure 4: House Image (a) Original image (b) HE processing (c) BBHE processing (d) RSWHE processing (e) AGCWD processing (f) Proposed technique

Figure 5 shows enhancement results on F-16 image by different techniques. Results with different techniques from Figure 5(b)5(e) shows that enhancement is not better by these techniques. It can be seen clearly from figure 5(f) that the proposed method efficiently enhances contrast of image without degrading the original colors of images.

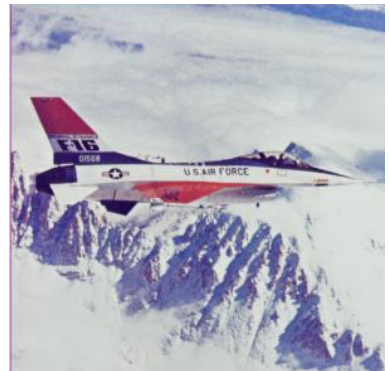

(a) Original Image

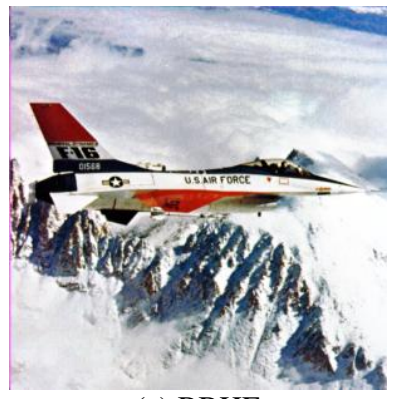

(c) BBHE

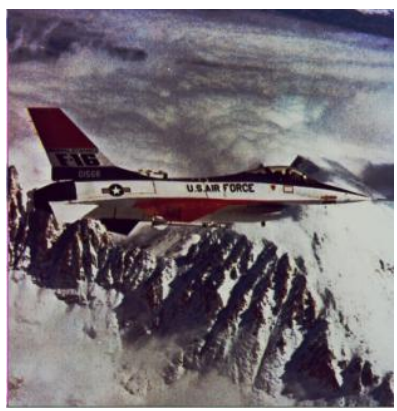

(e) AGCWD

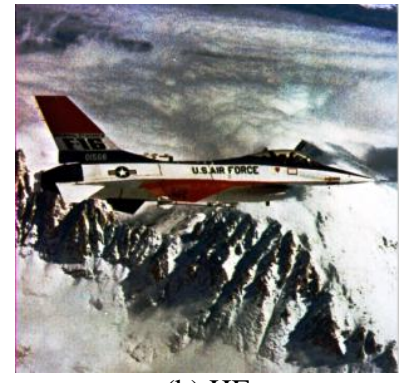

(b) HE

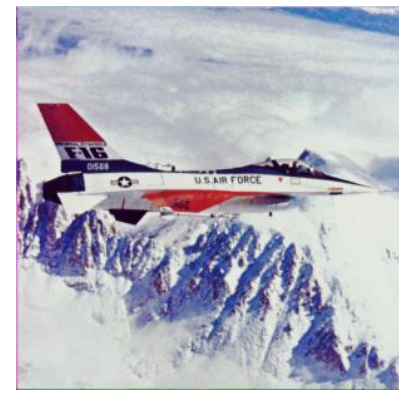

(d) RSWHE

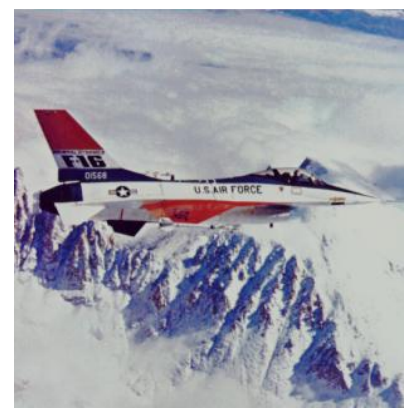

(f) Proposed Technique
Figure 5: F-16 Image (a) Original image (b) HE processing (c) BBHE processing (d) RSWHE processing (e) AGCWD processing (f) Proposed technique

\subsection{Performance measurement}

In this paper results are shown on a dataset of four images in which war plane image and cameraman image are gray scale images and house image and F-16 image are color images. The value of recursion is taken as 2 for proposed technique for a fair comparison. PSNR values comparison for all techniques on dataset images is shown in Table 1. MSE values comparison for all techniques on dataset images is shown in Table 2. AMBE values comparison for all techniques on dataset images is shown in Table 3.

Results are evaluated using widely used parameters PSNR (Peak Signal to Noise Ratio), MSE (Mean Square Error) and AMBE (Absolute Mean Brightness Error).

\subsubsection{Mean Square Error}

Let $\mathrm{X}(\mathrm{i}, \mathrm{j})$ be the input image and $\mathrm{Y}(\mathrm{i}, \mathrm{j})$ be the final image. Let there be $\mathrm{N}$ toatal number of pixels in input and output image, then Mean Square Error (MSE) is calculated as:

$$
\operatorname{MSE}=\sum_{i} \sum_{j}|X(i, j)-Y(i, j)|^{2} / \mathrm{N}
$$

Less the MSE value, the better will be the image quality [10]. 


\subsubsection{Peak Signal to Noise Ratio}

For input image $\mathrm{X}(\mathrm{i}, \mathrm{j})$ and output image $\mathrm{Y}(\mathrm{i}, \mathrm{j})$ Peak Signal to Noise Ratio is calculated as:

$$
\text { PSNR }=10 \log _{10}(\mathrm{~L}-1)^{2} / \mathrm{MSE}
$$

where $\mathrm{L}$ is the maximum number of gray levels. Greater value of PSNR means better image quality [10]

\subsubsection{Absolute Mean Brightness Error}

This parameter Absolute Mean Brightness Error (AMBE) is used to measure the change in original brightness of image. Let $\mathrm{X}_{\mathrm{m}}$ be the mean of input image and $\mathrm{Y}_{\mathrm{m}}$ is the mean of output image. Then AMBE is calculated as:

$$
\operatorname{AMBE}=\left|X_{m^{-}} Y_{m}\right|
$$

The less is the value of AMBE, more is the brightness preserved [10].
The contrast enhancement can be observed by PSNR values. PSNR values for different techniques on dataset of 4 images are shown in TABLE 1. Greater the PSNR value better will be the image quality. It can be observed from TABLE 1 that PSNR values for grayscale images is higher in proposed technique and in color images he results are slightly less than RSWHE technique, this is because of the color map. Results of proposed technique are better from AGCWD technique as the proposed technique is an improvement over AGCWD technique.

The brightness preservation can be estimated from AMBE parameter. AMBE values for different techniques on dataset of 4 images are shown in TABLE 3. Brightness is better preserved in proposed technique in case of grayscale images. In case of color images brightness is preserved better than AGCWD technique.

TABLE 1: Comparison of PSNR values

\begin{tabular}{|c|c|c|c|c|c|}
\hline Images & HE & BBHE & RSWHE & AGCWD & $\begin{array}{c}\text { Proposed } \\
\text { Technique }\end{array}$ \\
\hline War plane image & 58 & 61 & 69 & 59 & 71 \\
\hline $\begin{array}{c}\text { Cameraman } \\
\text { image }\end{array}$ & 67 & 66 & 68 & 69 & 72 \\
\hline $\begin{array}{c}\text { House } \\
\text { image }\end{array}$ & 66 & 66 & 73 & 66 & 72 \\
\hline $\begin{array}{c}\text { F-16 } \\
\text { image }\end{array}$ & 59 & 68 & 77 & 58 & 69 \\
\hline
\end{tabular}

TABLE 2: Comparison of MSE values

\begin{tabular}{|c|c|c|c|c|c|}
\hline Images & HE & BBHE & RSWHE & AGCWD & $\begin{array}{c}\text { Proposed } \\
\text { Technique }\end{array}$ \\
\hline $\begin{array}{c}\text { War plane } \\
\text { image }\end{array}$ & 0.0949 & 0.0465 & 0.0090 & 0.0857 & 0.0056 \\
\hline $\begin{array}{c}\text { Cameraman } \\
\text { image }\end{array}$ & 0.0127 & 0.0154 & 0.0099 & 0.0081 & 0.0042 \\
\hline House image & 0.0217 & 0.0178 & 0.0035 & 0.0165 & 0.0042 \\
\hline $\begin{array}{c}\text { F-16 } \\
\text { image }\end{array}$ & 0.0764 & 0.0115 & 0.0014 & 0.0932 & 0.0079 \\
\hline
\end{tabular}

TABLE 3: Comparison of AMBE values

\begin{tabular}{|c|c|c|c|c|c|}
\hline Images & HE & BBHE & RSWHE & AGCWD & $\begin{array}{c}\text { Proposed } \\
\text { Technique }\end{array}$ \\
\hline $\begin{array}{c}\text { War plane } \\
\text { image }\end{array}$ & 47.825 & 1.463 & 12.554 & 54.420 & 9.043 \\
\hline $\begin{array}{c}\text { Cameraman } \\
\text { Image }\end{array}$ & 9.345 & 24.258 & 13.239 & 4.540 & 1.611 \\
\hline House image & 12.976 & 13.999 & 4.233 & 23.123 & 12.123 \\
\hline $\begin{array}{c}\text { F-16 } \\
\text { image }\end{array}$ & 54.343 & 2.404 & 1.408 & 71.993 & 22.084 \\
\hline
\end{tabular}

\subsection{Graphical analysis}

Graphical analysis of results is shown below. Figure 6 shows graph for PSNR values for proposed technique and various other techniques. 


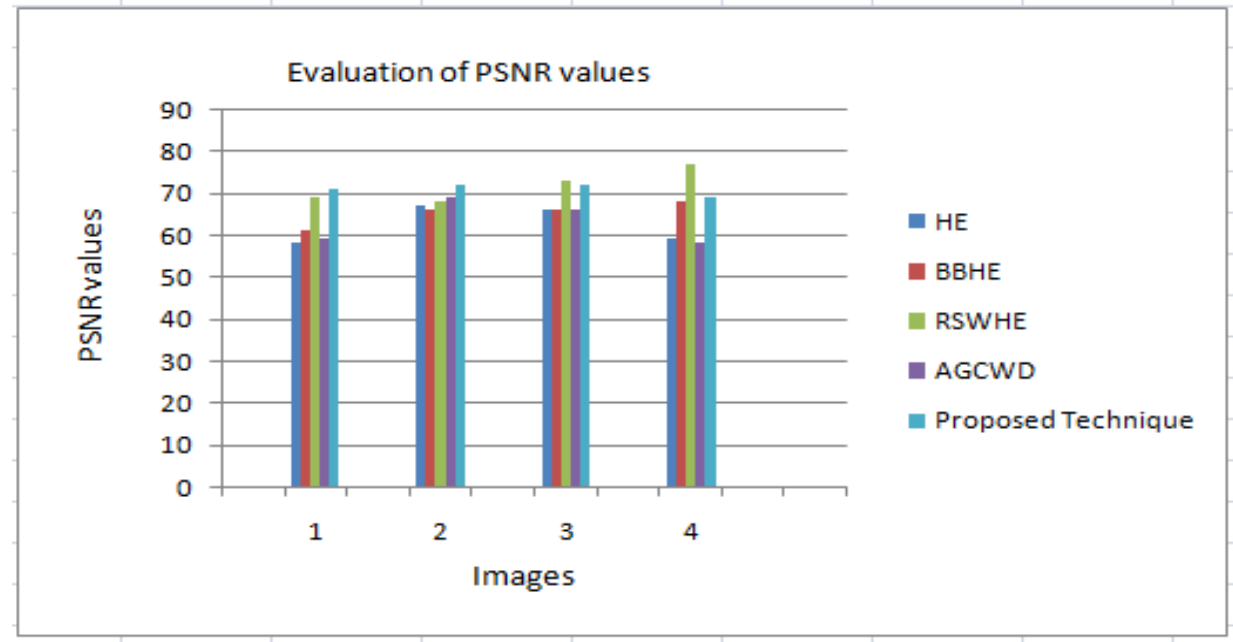

Figure 6: Evaluation of PSNR values

Figure 7 shows graph for MSE values for proposed technique and various other techniques.

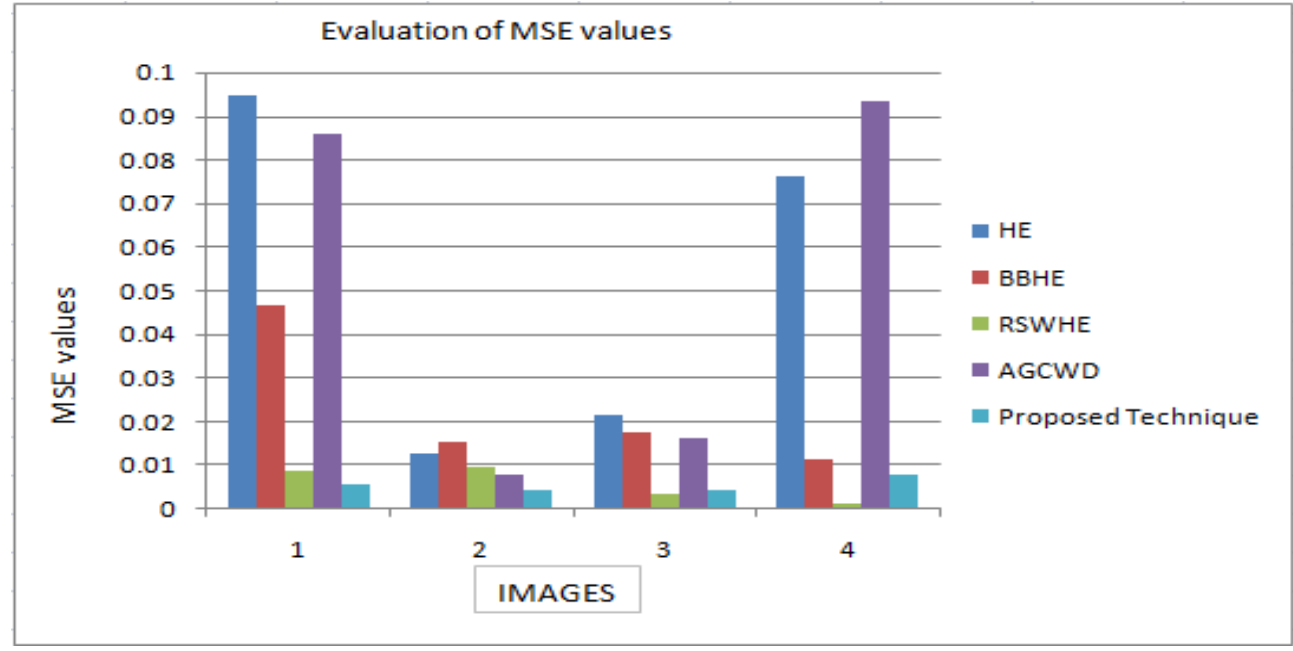

Figure 7: Evaluation of MSE values

Figure 8 shows graph for AMBE values for proposed technique and various other techniques.

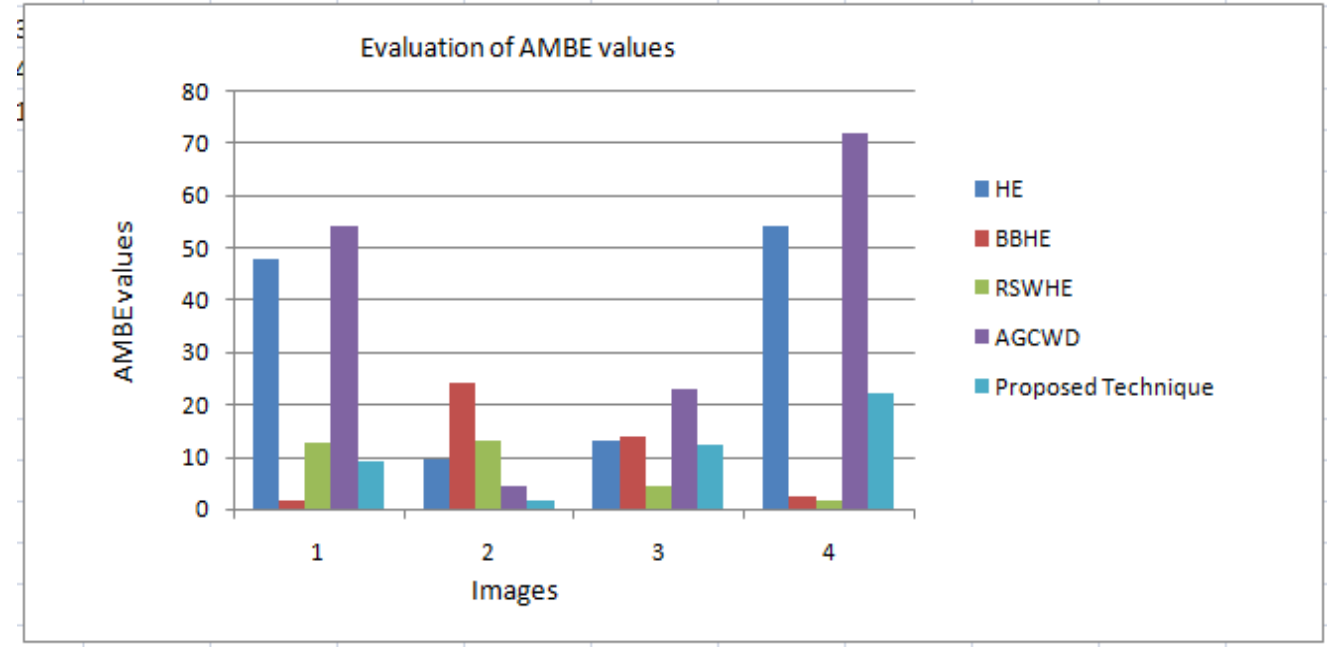

Figure 8: Evaluation of AMBE values 


\section{CONCLUSION}

Histogram equalization based contrast enhancement techniques are widely implemented. In this work also histogram equalization based technique is implemented. In this work recursive segmentation of histograms is done and then weighting method is applied to smooth down histogram. Further gamma correction is also applied that helps to improve brightness of image. The work improves the Adaptive Gamma Correction using Weighted Distribution (AGCWD) technique that had implemented Gamma Correction and Weighted Distribution techniques with no segmentation of histogram. Thus this work improves results by recursive segmentation of histograms that helps in better enhancement of images.

Further the work can be done to automate the recursion value according to the image, so that an appropriate value of recursion level can be used to segment histogram. Work can also be done to apply this technique on videos.

\section{REFERENCES}

[1] R. He, S. Luo, Z. Jing and Y. Fan, "Adjustable Weighting Image Contrast Enhancement Algorithm and its Implementation," IEEE Conference on Industrial Electronics and Applications, pp. 1750-1754, 21-23 June 2011.

[2] R. C. Gonzalez and R. E. Woods, Digital Image Processing, Prentice Hall, Upper Saddle River, New Jersey, 2nd Edition, 2002.

[3] S. C. Huang, F. C. Cheng and Y. S. Chiu, "Efficient Contrast Enhancement using Adaptive Gamma Correction with Weighting Distribution," IEEE Transactions on Image Processing, Vol. 22, No. 3, pp. 1032-1041, March 2013.

[4] Y. T. Kim, "Contrast enhancement using brightness preserving bi-histogram equalization," IEEE Transactions On Consumer Electronics, Vol. 43, No. 1, pp.1-8, February 1997.

[5] Y. Wang, Q. Chen, B. Zhang, "Image enhancement based on equal area dualistic sub-image histogram equalization method," IEEE Transactions on Consumer Electronics, Vol. 45, No. 1, pp. 68-75, February 1999.

[6] S. D. Chen, A. R. Ramli, "Minimum mean brightness error bi histogram equalization in contrast enhancement," IEEE Transactions on Consumer Electronics, Vol. 49, No. 4, pp. 1310-1319, November 2003.
[7] S. D. Chen, A. R. Ramli, "Contrast enhancement using recursive mean-separate histogram equalization for scalable brightness preservation," IEEE Transactions on Consumer Electronics, Vol. 49, No. 4, pp. 1301-1309, November 2003,

[8] C. Wang, Z. Ye, "Brightness preserving histogram equalization with maximum entropy: a variational perspective," IEEE Transactions on Consumer Electronics, Vol. 51, No. 4, pp. 1326-1334, November 2005

[9] K. S. Sim , C. P. Tso, Y. Y. Tan, "Recursive sub image histogram equalization applied to gray scale images," Pattern Recognition Letters, Vol. 28, No. 10, pp. 12091221, july 2007.

[10] M. Kim, M. G. Chung, "Recursively separated and weighted histogram equalization for brightness preservation and contrast enhancement," IEEE Transactions on Consumer Electronics, Vol. 54, No. 3, pp. 1389-1397, August 2008.

[11] Z. G. Wang, Z. H. Liang, C. L. Liu, "A real time image processor with combining dynamic contrast ratio enhancement and inverse gamma correction for PDP," Displays, Vol. 30, Issue 3, pp 133-139, July 2009.

[12] T. Celik, T. Tjahjadi, "Contextual and variational contrast enhancement," IEEE Transactions on Image Processing, Vol. 20, No. 12, pp. 3431-3441, December 2011.

[13] S. Ashish, S. Rajeev, P. Yogadhar, "An exhaustive analysis on various foggy image enhancement techniques," International Journal of Advanced Research in Computer Science and Electronics Engineering, Vol. 3, No. 1, pp. 1117, January 2014.

[14] S. Mohanram, B. Aarthi, C. Silambarasan, T. Joyce Selva Hephzibah, "An optimized image enhancement of foggy images using gamma adjustment," International Journal Of Advanced Research In Electronics And Communication Engineering, Vol. 3, No. 2, pp. 155-159, February 2014.

[15] R. Chauhan, S. S. Bhadoria, "An improved image contrast enhancement based on histogram equalization and brightness preserving weight clustering histogram equalization," International Conference on Communication Systems and Network Technologies, pp. 597-600, 3-5 June 2011.

[16] O. Marques, Practical Image and Video Processing using MATLAB, John Wiley and Sons, Hoboken, New Jersey, 1st Edition, 2011. 